\title{
Current Concepts in the Treatment of Patellofemoral Instability
}

\author{
Gary Ulrich, B.S. ${ }^{1}$ and Hemant Pandit, FRCS (Orth), DPhil (Oxon) ${ }^{2}$ \\ ${ }^{1}$ Indiana University School of Medicine, ${ }^{2}$ University of Leeds, Leeds Institute of \\ Rheumatic and Musculoskeletal Medicine
}

\section{Background and Hypothesis:}

Patellofemoral instability represents a disabling condition, which presents primarily in active, young patients with an increased incidence in the female sex. A patellar dislocation can occur from a high-energy trauma or from an atypical anatomy, such as trochlear dysplasia, patellar dysplasia, patella alta, increased tibial tuberosity-trochlear groove (TT-TG) distance, increased Q-angle, and ligamentous laxity. When a patient presents with patellofemoral instability, the orthopedic surgeon faces many decisions regarding the treatment. Since the risk of a second dislocation after an acute dislocation resides at approximately $17 \%$, many orthopedic surgeons treat the first patellar dislocation non-operatively barring the patient lacks any atypical anatomy. However, after a second dislocation, the likelihood of recurrent dislocations increases to approximately $50 \%$, which directs most orthopedic surgeons to surgical treatment. The current work reviews the anatomical predispositions, clinical presentation, and treatment of patellofemoral instability.

\section{Experimental Design or Project Methods:}

The review was constructed via an extensive literature search utilizing the databases of MEDLINE/PubMed, SportDiscus, CINAHL, and Cochrane Central Register of Controlled Trials.

\section{Results:}

Multiple treatments exist for patellofemoral instability, which include nonoperative treatment, MPFL reconstruction, tibial tubercle osteotomy, and trochleoplasty, along with many other procedures. Each case requires a tailored approach to successfully treat the patellofemoral instability.

\section{Conclusion and Potential Impact:}

Patellofemoral instability represents a potentially debilitating condition of anterior knee pain and limited activity. Understanding the anatomical predispositions, clinical presentation, and treatment of patellofemoral instability marks the first step to caring for patients with this condition. 